\title{
SIMONIC: IoT Based Quarantine Monitoring System for Covid-19
}

\author{
Vita Awalia Mardiana a, *, Mochamad Mardi Marta Dinata a, Galih Nugraha \\ Nurkahfi a , Arumjeni Mitayani a, Dayat Kurniawan a , Nasrullah Armi a, b, Budi \\ Prawara $^{a}$, Sudirja Sudirja ${ }^{c}$, Andria Arisal ${ }^{\mathrm{d}}$, Rendra Dwi Firmansyah ${ }^{\mathrm{e}}$, Andri \\ Fachrur Rozie $^{d}$, Sulaksono Priyo ${ }^{\text {a }}$, Sopyan Setiana ${ }^{\text {a }}$, Asih Setiarini ${ }^{\text {a }}$ \\ ${ }^{a}$ Research Center for Electronics and Telecommunication \\ National Research and Innovation Agency \\ Bandung, Indonesia \\ ${ }^{b}$ Telecommunication Engineering, School of Electrical Engineering \\ Telkom University \\ Bandung, Indonesia \\ ${ }^{c}$ Research Center for Electrical Power and Mechatronics \\ National Research and Innovation Agency \\ Bandung, Indonesia \\ ${ }^{d}$ Research Center for Informatics \\ National Research and Innovation Agency \\ Bandung, Indonesia \\ e Technical Implementation Unit for Instrumentation Development \\ National Research and Innovation Agency \\ Bandung, Indonesia
}

\begin{abstract}
COVID-19, which has become a global pandemic since March 2020, has tremendously affected human life globally. The negative impact of COVID-19 affects societies in almost all aspects. Implementing quarantine monitoring, also social distancing, and contact tracing are a series of processes that can suppress the new infected COVID-19 cases in various countries. Prior works have proposed different monitoring systems to assist the monitoring of individuals in quarantines, as well as many methods are offered for social distancing and contact tracing. These methods focus on one function to provide a reliable system. In this paper, we propose IoT-based quarantine monitoring by implementing a geofence equipped with social distancing features to offer an integrated system that provides more benefits than one system carrying one particular function. We propose a system consisting of a low cost, low complexity, and reusable wristband design and mobile apps to support the quarantine monitoring system. For the geofencing, we propose a GPS-based geofence system that was developed by taking advantage of the convenience offered by the Traccar application. Meanwhile, we add the notification for social distancing feature with adaptive distance measurement RSSIbased set up in the android application. Based on the experiment we did to validate the system, in terms of wristband-to-smartphone communication, scanning interval in smartphone and advertising interval in wristband is best to set in $7 \mathrm{~s}$ for both. For social distancing notification and geofence, we measure the system performance through precision, recall, accuracy, and F-measure.
\end{abstract}

Keywords: IoT-quarantine monitoring system, IoT-social distancing system, Traccar-based geofence, SIMONIC, low-cost wristband.

\section{INTRODUCTION}

COVID-19 (Corona Virus Disease 2019), which has become a global pandemic since March 2020, has tremendously affected human life globally [1]. The negative impact of COVID-19 affects societies in almost all aspects. All the authorities and governments working together with scientists from various backgrounds and disciplines propose many methods, rules, and set policies to defeat the difficulties due to COVID-19. The world was able to suppress the rate of viral infections in early 2021. Experts are working hard to accelerate research so

\footnotetext{
* Corresponding Author.

Email: vitaawalia@gmail.com

Received: November 30, 2021 ; Revised: December 18, 2021

Accepted: December 25, 2021 ; Published: December 31, 2021

Open access under CC-BY-NC-SA

(C) 2021 PPET - LIPI
}

that vaccines with high efficacy levels can be immediately produced in large quantities to be distributed to the public. However, in its development, this virus undergoes multiple mutations, spreading faster than the previous variant, again increasing the world infection rate [1], so it still requires hard work from various parties to get through this problem together.

Implementing quarantine monitoring, also social distancing, and contact tracing are a series of processes that can suppress the new infected COVID-19 cases in various countries, such as in [2]-[3]. Prior works have proposed different monitoring systems to assist the monitoring of individuals in quarantines [4]-[5], as well as many methods, are offered for social distancing and contact tracing [6]-[10]. These methods focus on one function to provide a reliable system. Meanwhile, this paper proposes IoT (Internet of Things)-based quarantine 
monitoring by implementing a geofence also equipped with social distancing features to offer an integrated system to provide more benefits than one system carrying one particular function.

Previous proposed individual monitoring systems for COVID-19 quarantine can be classified into three categories based on the supporting components. There are smartphone-based [4], wristband global positioning system (GPS) [11], wristband connected to a smartphone [5], [12]. Each monitoring system mentioned has different capabilities, with the drawbacks and benefits associated according to the usage of its supporting components. GPS wristband-based monitoring system requires an accurate GPS module to provide position estimation with minimum error. This design tends to be in a medium system complexity related to high power consumption and cost. Moreover, the production process is not simple, and there is no room for wristband function development when mass-produced. On the other hand, the monitoring system relying on smartphone apps is prone to provide unreliable position data due to the low possibility of smartphones being carried all the time by individuals in the quarantine process.

To build a system that can provide good reliability data of patient position, we proposed a system employing wristband and mobile apps compared to other systems. This configuration also gives the flexibility to add the required applications installed in the smartphone. Such applications include patient registration and database for associated data needed, individual check of the working monitoring system, integrated web for supervisors in conducting quarantine supervision, and the possibility of adding other features in future product developments. A non-removable-attached wristband to the user body connected all the time to smartphone apps accommodates better patient position monitoring during quarantine. In this research, we design a low cost, low complexity, and reusable design of wristband and android-based mobile apps. This system is called SIMONIC (Smart Innovated Monitoring for Covid-19).

Geofencing is the technology used for many purposes such as for activity recognition for dementia/Alzheimer patients [13]-[14], road safety [15], HIV transmission prevention [16], and also researcher implements it for quarantine monitoring movement of COVID-19 patients [4]-[5], [12]. Signature learning technology can learn the shape of the room by performing a learning process in the quarantine procedure is first carried out in [5]. The system then sends the size of the quarantine room to the server and makes that room dimension a geofence. Research that developed an application to create geofences based on GPS readings can be found in [13]. Study about GPS-based use for the geofence process and supported by AI (Artificial Intelligence) for face recognition can be found in [4]. Lastly, the application is able to send notifications when the patient/user exits the pinpoint as far as $100 \mathrm{~m}$ and above is studied in [12]. In contrast to some of the applications that have been made, in this paper, we propose a GPS-based geofence system that was developed by taking advantage of the convenience offered by the Traccar application. Traccar is an open- source tracking system that supports more than 130 types of communication protocols. By using Traccar, the process of making monitoring applications becomes easier.

Previous research proposed several kinds of social distancing methods. The various algorithms used are AIbased, for example, study about deep learning methods to process video data and applying object detection to be able to calculate the approximate distance of several people in a place with the help of a graphic processing unit (GPU) [6], [17]-[18]. The same method is discussed, but it was applied to robots [19]. The following social distancing algorithm is GPS-based, which used an application on a smartphone [7]. This paper utilizes inverse perspective mapping (IPM) with the camera's intrinsic information in producing a bird's eye view with real-world coordinates of the frame being processed from a video source [20]. Lastly, the method of social distancing using ultrasonic sensors is discussed in the paper [21]. Each of these systems has its advantages and disadvantages in terms of system complexity, price, power consumption, system accuracy, and whether it is applicable or not for public usage. To optimize the SIMONIC system without adding other components, we utilize the received RSSI (Received Signal Strength Indicator) value from the BLE beacon wristband signal as a reference for distance measurement and propose an adaptive distance measurement method.

To create the wristband design, the complex design and cost are considered. Previous wristband design has to provide GPS and GSM modules [11], and another design that included the microcontroller needs to be preprogrammed [12]. Compared to them, SIMONIC wristband designs are much more straightforward in terms of module used and module programming. The system complexity of the SIMONIC's wristband is made as low as possible by eliminating components on the device, which are not directly needed to support the operational monitoring of individuals who are undergoing self-quarantine such as positioning service and GSM module communication which already provide by smartphone. The SIMONIC's wristband main component, as can be seen in Table 1, is HM-10 to provide a BLE beacon signal. The main component price of SIMONIC's wristband is lower than the main component of the previous two designs mentioned earlier.

SIMONIC proposes a wristband-mobile apps system to conduct individual monitoring. The geofence feature of SIMONIC is created using the Traccar application, and the adaptive social distancing feature is set up in the android application. Furthermore, the wristband is created in low cost, low complexity, and reusable design. In this study, we conducted three main experiments: a) empirical studies on the performance of BLE communication between SIMONIC wristbands and several types of smartphones in the spatial aspect (quarantine room) and also the durability of the wristband battery; b) the performance of the distance measurement method that we propose for SIMONIC and several features of the SIMONIC application; and c) the performance of the geofence system evaluation. 


\section{System Design AND Methodology}

\section{A. System Design}

Figure 1 represents the system design of SIMONIC. There are two main parts: client and server. On the clientside, individuals in the quarantine period wear SIMONIC wristbands that emit beacon signals in a specific advertising interval. A smartphone with a SIMONIC application installed captures the beacon signal containing the universally unique identifier (UUID). The mobile apps then send UUID data and location coordinates to the server via cellular communication and internet lines. The smartphone and wristband communication uses the Bluetooth low energy (BLE) communication protocol. At the beginning of the quarantine period, we need a registration process to pair one wristband with one mobile app. This process is intended for initial data collection of quarantine patients by officers.

Furthermore, the other purpose is to calibrate SIMONIC systems in different smartphones and rooms. Individuals also fill in personal data such as name, personal identity number, residential address, and quarantine region in this registration process. The smartphone GPS sensor needs to be continually activated to access the quarantine location's location coordinates.

While the server-side consists of a server for data processing and a web for data visualization. Some of the server functions: a database for quarantine participant data, including wristband number and smartphone position coordinates, geofence setting, monitor and display quarantine participant data in real-time whether they stay at the quarantine location or not, and provide alarms when a violation occurs in the quarantine process. This part of the server is managed by the government, which authorizes conducting quarantine monitoring, either centrally or vice versa.

On the other hand, the wristband is designed as low cost, low complexity, and reusable. It is made from local material. The straps are made from rubber, the electronic part composed of BLE chip to transmit beacon signal and li-batt which will provide sufficient power for 14 days quarantine. Table 1 explains the specification of the wristband.

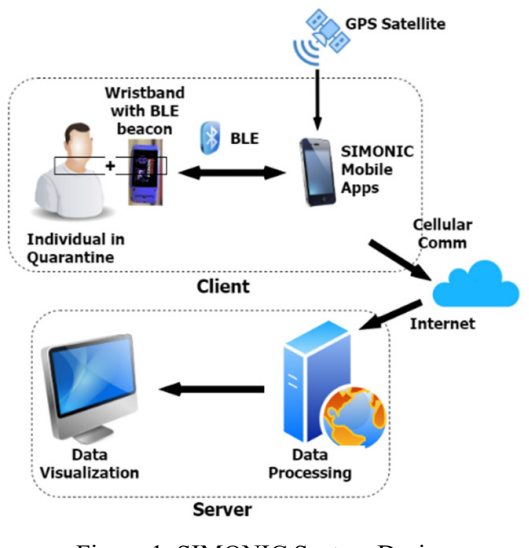

Figure 1. SIMONIC System Design.
TABLE 1

WRISTBAND COMPONENT

\begin{tabular}{|c|l|l|}
\hline No & Wristband Part & \multicolumn{1}{c|}{ Component } \\
\hline 1 & Wristband strap & Rubber \\
\hline 2 & BLE component & HM-10 \\
\hline 3 & Li-Batt & Lithium-Battery PL582728 \\
\hline 4 & Cable connector & Copper Cable $(0.65 \mathrm{~mm})$ \\
\hline 5 & Screw & M2x10 \\
\hline 6 & Magnet Connector & Magnet Connector Male \& Female \\
\hline
\end{tabular}

\section{B. Proposed Method for Distance Measurement} (Notification for Social Distancing)

On the client-side, this monitoring system consists of a wristband and smartphone that maintains their communication to provide the location of quarantine participants in a dedicated room. Besides the geofence feature, this system is also equipped with a notification for social distancing feature to provide alerts if a confirmed COVID-19 positive patient wearing a SIMONIC wristband is accidentally approached. Therefore, the system must estimate the distance measurement from the smartphone to the wristband as precisely as possible to carry out its function optimally. In this system, we employed power regression to convert RSSI value to distance value.

Power regression is a method used to study the relationship between one variable and another, for example, $\mathrm{x}$ and $\mathrm{y}$. If there is an independent variable $\mathrm{x}$ whose value is known, we can find the dependent variable $\mathrm{y}$, resulting from the value of $\mathrm{x}$ behavior. Previous studies have used power regression for distance measurement in IoT systems employing BLE/WiFi (Wireless Fidelity) communication technology with RSSI as a reference value [7]. The power regression formula for calculating distance is represented in (1). We need the RSSI reading in 1 meter as a reference to get this constant value.

$$
Y=A\left(\frac{r}{x}\right)^{B}+C
$$

Where $\mathrm{Y}$ is the distance in meters, $\mathrm{r}$ is RSSI measured by the device, $\mathrm{x}$ is the reference RSSI value at 1 meter, $\mathrm{A}$, $\mathrm{B}$, and $\mathrm{C}$ are constants used to minimize loss factors resulting from signal interference.

Apart from hardware problems and the transceiver antenna's sensitivity, one thing that causes the RSSI value to be spiky in indoor measurement is multipath fading. The signal interference varies according to the room layout or surface material structure. This interference is unique according to the type of room occupied. The RSSI value of the BLE signal measurement results in room A may be different when compared to the measurement results in room B using the same type of measuring instrument. Furthermore, individuals have different types of smartphones and carry different qualities of receiver sensitivity influenced by the chipset, antenna layout, or OS (Operating System) configuration.

We proposed an adaptive distance measurement algorithm, described in Figure 2, to accommodate the quarantine needs. The calibration process is carried out in the registration process to obtain the ground truth of the one-meter RSSI measurement value. To validate the 


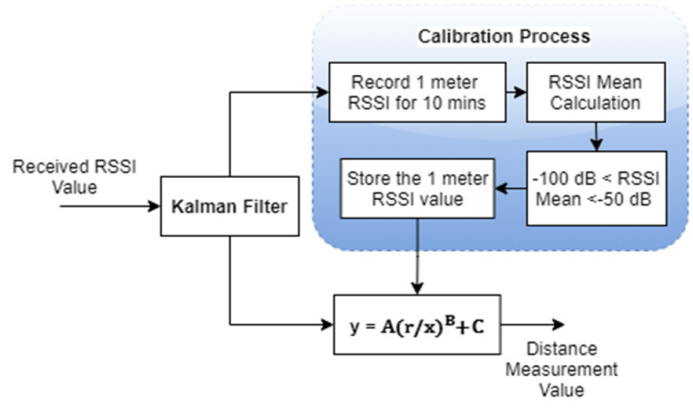

Figure 2. Distance Measurement Algorithm.

calibration process, we check the results of the RSSI calibration whether it is in the range of $-100 \mathrm{dBm}$ until $50 \mathrm{dBm}$ or not (this value is defined from the empirical study discussed in chapter III.B, and we took the range of the highest and lowest RSSI values from the study). If the RSSI value of the calibration results is in that range, the calibration process is validated, and the value is saved as a reference value for calculating distances.

\section{Geofence Design for Monitoring Purpose}

For an easy and fast application development process, we developed individual monitoring with geofence application based on Traccar. Traccar is an open-source modern GPS tracking platform that is developed innovatively and securely [22]. Traccar provides several features: a live tracking function to monitor GPS devices under supervision, instant web notifications through email and SMS (Short Message Service), simple location history, trips, charts, and summary reports that can be viewed directly on the web or downloaded in excel format [22]. Traccar also supports the creation of geofence zones in three geometry types: circle, polygon and polyline, and can generate events to enter and exit the geofence zone.

Generally, the Traccar-based geofence setting is depicted in Figure 3. This geofence is set during the registration process. The handphone needs to be placed in the center of the room for 5 minutes to set the geofence. The application will detect the geographical coordinates obtained from the mobile phone participant's GPS. Then the supervisor can choose the geofence type per quarantine condition and location, either determined-size or custom-size. Determine-size is accommodated using built-in geofence in apps in a circle shape with a radius of $10 \mathrm{~m}$, a rectangular shape of $10 \mathrm{~m} \times 5 \mathrm{~m}$, and a medium rectangular shape of $6 \mathrm{~m} \times 4 \mathrm{~m}$. The supervisor can create custom geofence size as per the requirement of the actual

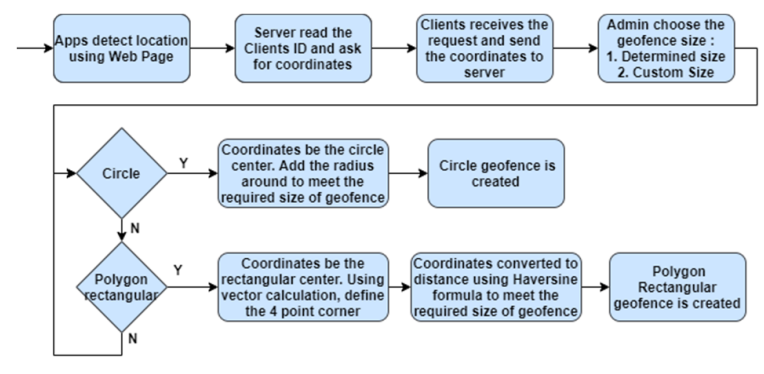

Figure 3. Traccar-based Geofence Setting. size of the quarantine location, which a built-in geofence cannot accommodate.

\section{EXPERIMENTAL SCENARIO AND RESULT}

In order to validate this system utility, we have performed three experimental scenarios: 1) empirical studies of BLE communication and wristband battery durability; 2) notification for social distancing performance; and 3) geofence performance.

\section{A. Empirical Study of BLE Communication}

In this part, we perform an empirical study to measure the capability of communication performance between smartphones and wristbands. We conduct measurement in two steps. Firstly, we measure the communication performance in line-of-sight (LOS) indoor and outdoor areas from 1 to $20 \mathrm{~m}$, as represented in Figure 4. Furthermore, in the second step, we measure the communication performance in some size of quarantine rooms represented in Figure 5.

For the first step, we measure the RSSI attenuation and communication performance synchronization related to distance in LOS indoor and outdoor areas. We measure the RSSI value for 5 minutes at each distance. As shown in Figure 6, for LOS outdoor vs. indoor conditions, the RSSI value decreases significantly around $35 \mathrm{~dB}$ to 40 $\mathrm{dB}$ from $1 \mathrm{~m}$ to $5 \mathrm{~m}$ distance. Meanwhile, from $5 \mathrm{~m}$ to 20 $\mathrm{m}$, The RSSI value decreases around $10 \mathrm{~dB}$ in indoor and outdoor areas. In the outdoor area, the variation of RSSI values is quite the same in each distance we measure, as represented by boxplot size. While in the indoor environment, the most considerable RSSI variation is in the $1 \mathrm{~m}$ distance, and the lowest RSSI variation is in the $20 \mathrm{~m}$ distance.

Besides, we measure the BLE communication synchronization performance in LOS indoor and outdoor for 10 minutes. As we can see in Figure 7, notice that the increment of range decreases the communication synchronization performance. The decrement of communication synchronization performance is also caused by the searching interval mode used in this system. The searching interval is the interval time of the smartphone to scan the BLE signal emitted by the wristband, which is set to be continuous, one scanning per $3 \mathrm{~s}$, and one scanning per $7 \mathrm{~s}$. The overall value of outdoor synchronization is slightly higher than the value of indoor synchronization. It can be affected by multipath

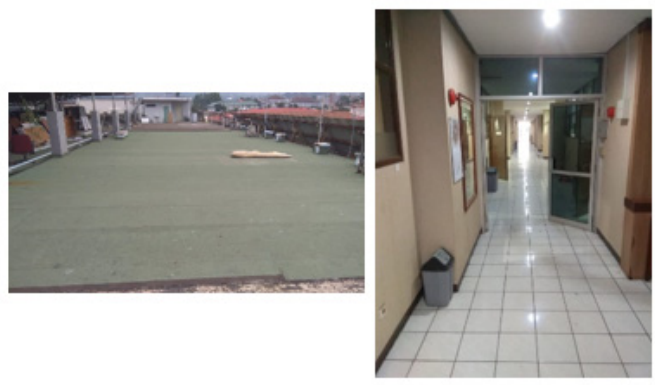

(a)

(b)

Figure 4. LOS Area for SIMONIC Measurement. (a) Outdoor and (b) Indoor. 


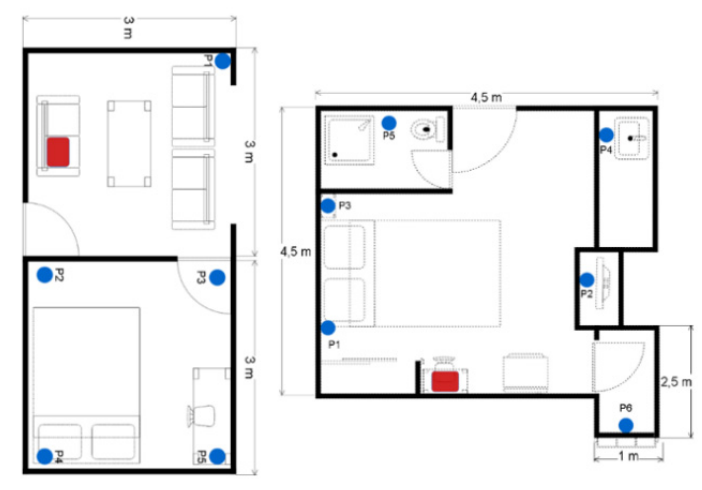

(a)

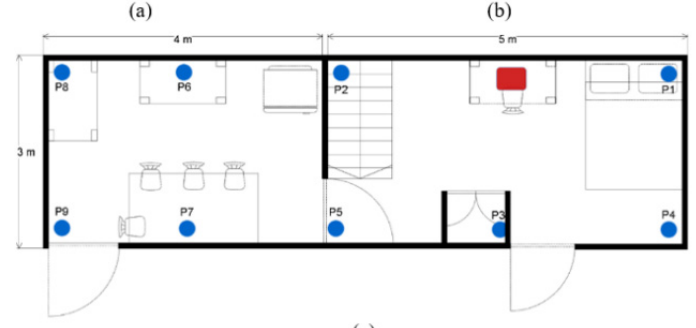

(c)

Figure 5. Various Quarantine Rooms: (a) Small Room $\left(18 \mathrm{~m}^{2}\right)$, (b) Apartment Room $\left(24 \mathrm{~m}^{2}\right)$, (c) Pavilion Room $\left(27 \mathrm{~m}^{2}\right)$.

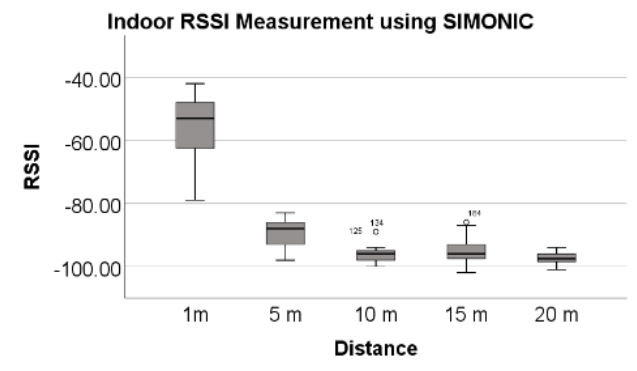

(a)

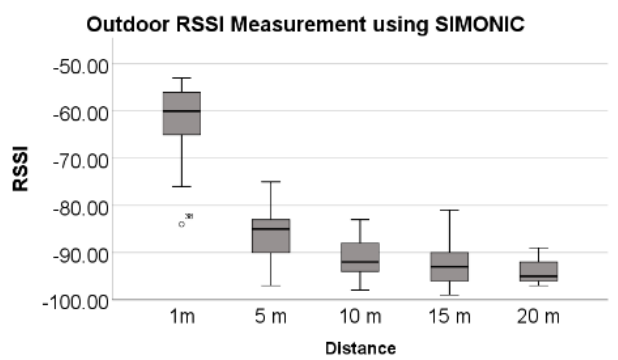

(b)

Figure 6. RSSI vs. Distance of SIMONIC Measurement in LOS Area (a) Indoor and (b) Outdoor.

fading, which happens more in indoor rather than in outdoor areas.

For the second step, we measure the RSSI value in various quarantine room sizes and conditions. Here we measure the performance of SIMONIC communication inside three different rooms. Firstly, it is in the small room $\left(18 \mathrm{~m}^{2}\right)$, secondly is in the apartment $\left(24 \mathrm{~m}^{2}\right)$ and thirdly is in the pavilion room $\left(27 \mathrm{~m}^{2}\right)$. The room size and condition are depicted in Figure 5(a), (b) and (c), respectively. We use four different smartphones in this measurement, and the result is depicted in Figure 8(a),

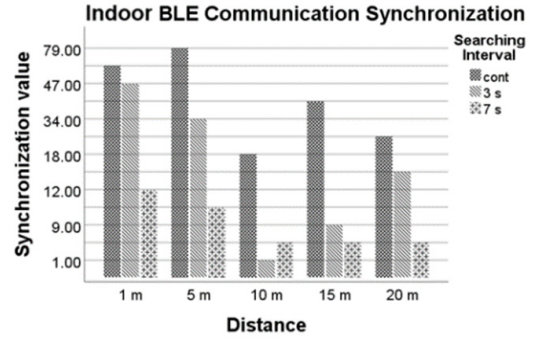

(a)

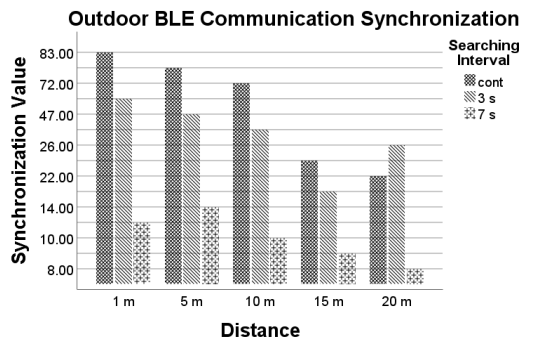

(b)

Figure 7. Synchronization vs. Distance of SIMONIC Measurement in LOS area. (a) Indoor and (b) Outdoor.

(b), and (c). We measure the RSSI value for 5 minutes at each distance.

We can see that each smartphone read a pretty diverse mean of RSSI in a room, with the same environment and possibility of the same multipath fading. So, the device performance is the most significant factor that creates different RSSI reading values. From these measurements, we can conclude that the Note $10+$ can read the highest RSSI value in all rooms among all smartphones, and the Mi 10 read the lowest RSSI value in all rooms. Overall, the farther the wristband from the smartphones, the smaller the RSSI value we get.

In the small room, communication between the wristband and the smartphones is successful in all the measurement points we mark with blue points. However, communication is not successful in measurement points 6 in the apartment room and 9 in the pavilion room. A wall barrier creates a high resistance value to the SIMONIC system.

Next, we measure wearable device battery durability with the following methods:

1) The specification of the battery used is a lithium battery with a model PL582728 with a capacity of $3500 \mathrm{mAh}$, operating voltage of $3.7 \mathrm{~V}$, and a cut-off voltage of $3.2 \mathrm{~V}$.

2) Configuration of battery settings combines advertising intervals $(100 \mathrm{~ms} ; 4000 \mathrm{~ms} ; 7000 \mathrm{~ms})$ and power modules $(-23 \mathrm{dBm} ;-6 \mathrm{dBm} ; 0 \mathrm{dBm} ; 6$ $\mathrm{dBm}$ ) so that a total of 12 wearable devices will be measured simultaneously.

3) Measurements were carried out for 14 days following the recommendations for self-quarantine for COVID-19 patients by measuring the battery voltage using a multimeter seven times a day, namely every 05:00, 08:00, 11:00, 14:00, 17:00, 20:00, and 23:00 WIB.

4) At the beginning of use, the battery voltage is between $3.96 \mathrm{~V}$ to $4.05 \mathrm{~V}$. 


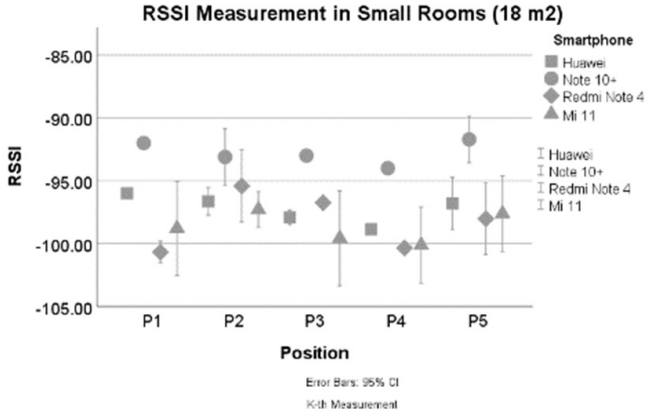

(a)

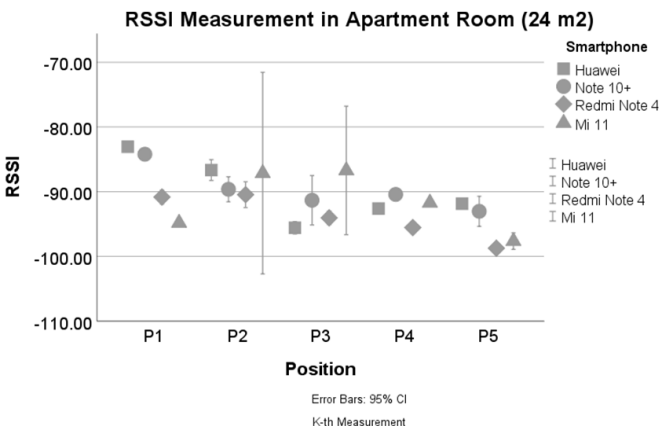

(b)

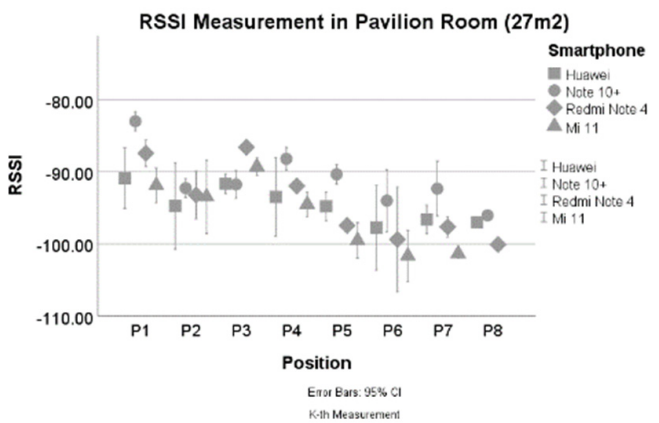

(c)

Figure 8. SIMONIC Measurement in Various Quarantine Rooms: (a) Small Room $\left(18 \mathrm{~m}^{2}\right)$, (b) Apartment Room $\left(24 \mathrm{~m}^{2}\right)$, (c) Pavilion Room $\left(27 \mathrm{~m}^{2}\right)$.

The results of measuring battery life on this wearable device can be seen in Figures 9(a), (b), and (c). Figure 9 shows that among the three advertising intervals tested, wearable devices with $100 \mathrm{~ms}$ intervals showed the fastest decrease in battery voltage compared to $4000 \mathrm{~ms}$ and $7000 \mathrm{~ms}$ intervals whose voltage was still stable until day 14. This happens because the information sent by the wearable device will increase a lot if the advertising interval is getting shorter. For example, in 60 seconds, a wearable device with an advertising interval of $7000 \mathrm{~ms}$ will only send information nine times, an advertising interval of $4000 \mathrm{~ms} 15$ times, while an advertising interval of $100 \mathrm{~ms}$ will send information 600 times.

When viewed from the power module configuration, the $6 \mathrm{dBm}$ power module configuration will consume the battery the fastest compared to other power modules. Next, to determine the power module used, we carry out continuous measurements of the RSSI BLE wearable device by moving between rooms while holding the measuring instrument. The results can be seen in Figure 9(d), where at the farthest distance from the measuring instrument, a wearable device with a $0 \mathrm{dBm}$ power

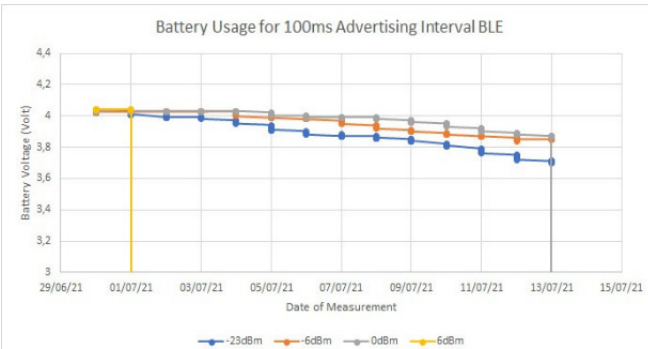

(a)

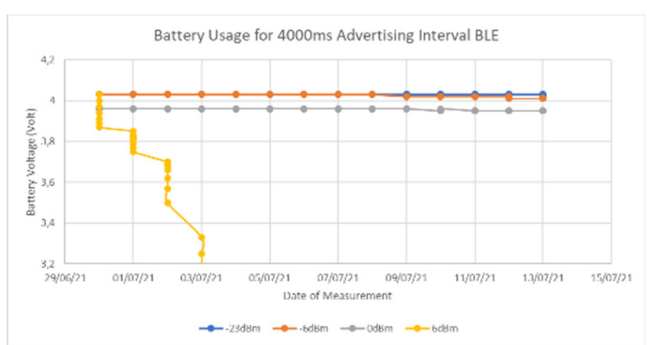

(b)

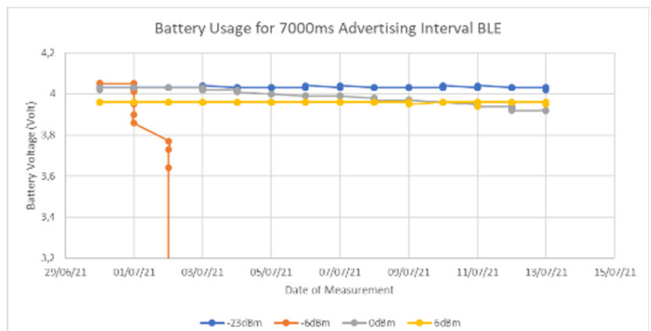

(c)

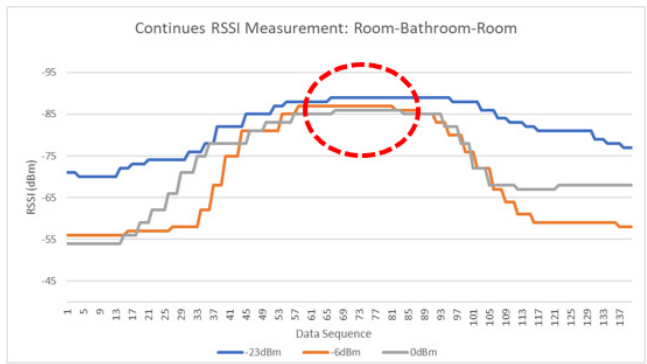

(d)

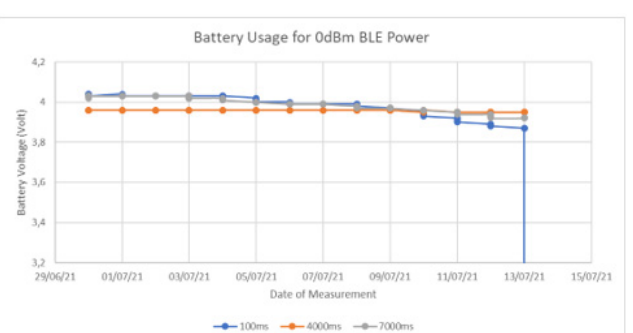

(e)

Figure 9. (a) BLE Battery Consumption with Advertising Interval 100 ms, (b) BLE Battery Consumption with Advertising Interval $4000 \mathrm{~ms}$, (c) BLE Battery Consumption with Advertising Interval $7000 \mathrm{~ms}$,

(d) Continues RSSI Measurement: Room-Bathroom-Room, (e) BLE Battery Consumption with Module Power $0 \mathrm{dBm}$.

module has the best RSSI value compared to $-6 \mathrm{dBm}$ and $-23 \mathrm{dBm}$.

Based on the measurement results, the power module applied to the wearable device is $0 \mathrm{dBm}$. Next, we measure battery life on wearable devices which use a 
$0 \mathrm{dBm}$ power module with a different combination of advertising intervals: $100 \mathrm{~ms}, 4000 \mathrm{~ms}$, and $7000 \mathrm{~ms}$. As shown in Figure 9(e), the battery voltage with an advertising interval of $100 \mathrm{~ms}$ will drop to a voltage of $1.74 \mathrm{~V}$. Meanwhile, for the $4000 \mathrm{~ms}$ and $7000 \mathrm{~ms}$ intervals, the battery voltage is more stable which is estimated to last more than 30 days.

\section{B. Distance Measurement Analysis (Notification for Social Distancing Performance)}

The distance calculation employing the power regression method requires an RSSI reference value at a one-meter apart, which cannot be generalized to all environments and conditions. As we know, smartphones with different brands and types have different receiver sensitivity levels. Each room has different physical characteristics resulting in different RSSI magnitudes reading in the same distance. Reflecting on the project carried out by The Opentrace [23], they conducted experiments of proximity detection measuring the RSSI value at 2-meter separation using various types of smartphones in the anechoic chamber. They achieve a wide range between -50 to -85 of the RSSI distribution results and utilize this value as the reference of the trace together system.

In this experiment, we conduct empirical studies to learn one-meter RSSI reading characteristics from the SIMONIC BLE wristband. We use a fixed transmit beacon interval at $7 \mathrm{~s}$ and transmit beacon power at 0 $\mathrm{dBm}$ as a wristband default setting. We employ several smartphones types with a BLE scanner installed to measure the RSSI. As shown in Figure 10, the same smartphone in different rooms yields quite different RSSI readings 1 meter apart, ranging from -95 to -65 . Compared to the RSSI from The Opentrace database, the RSSI variations read from the SIMONIC wristband are lower and diverse. Finally, based on our experiment, we define the lower and upper limits of RSSI reference for our calibration process ranging from -50 to -100 .

When the monitoring system is used in dynamic conditions, RSSI can quickly fluctuate. Therefore, we use the Kalman filter to get a smooth and stable RSSI value. The previous study has been clearly explained that the Kalman filter has a significant impact to smoothen the value of moving objects [24]-[25]. In this system, we proposed to use the Kalman filter, which we added from the android library. To evaluate the overall performance of the distance measurement method, we conducted

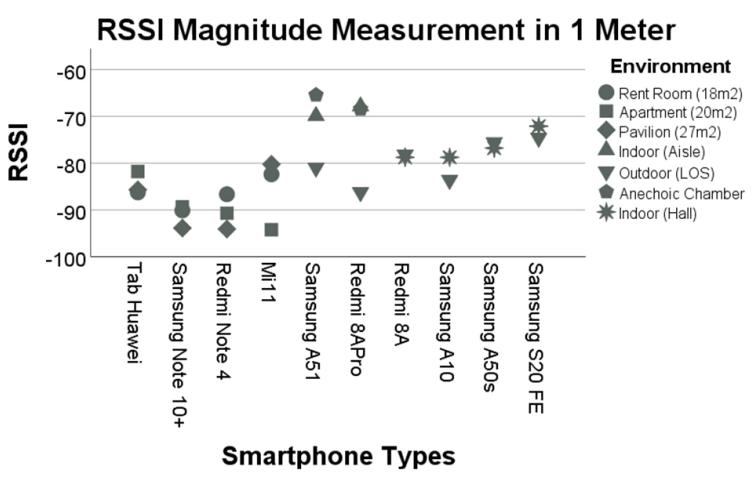

Figure 10. RSSI vs. Smartphone Types in the 1-meter Distance.

experiments in the LOS indoor and outdoor environments. We measure the distance read by the system from $0.25 \mathrm{~m}$ to $40 \mathrm{~m}$ of the actual distance, and the result can be seen in Figure 11. In the indoor environment, we conduct experiments in measurement points represented by blue points in the room. The results of RSSI reading under static conditions, repeated three times per location, are represented in Figure 12 (a), (b), and (c).

We also evaluate notification for social distancing performance. This experiment aims to measure the system's ability to recognize any SIMONIC wristband approaching a smartphone with SIMONIC apps installed. In the escape case of the patient from quarantine location and blend in the crowded place, any smartphone in the crowded area which has SIMONIC apps installed will give a notification for social distancing alert based on the distance set in the apps $(5 \mathrm{~m}, 10 \mathrm{~m}, 15 \mathrm{~m}, 20 \mathrm{~m})$. The performance evaluation is conducted in a crowded indoor and outdoor area that is expected to represent a real scenario in the field and is assessed by measuring the quantitative experiment result in precision, recall, accuracy, and F-1 Score. These four terms are the interpretation of system performance measures, which will be explained in the next paragraph. The experiment scenario is we set the scanning radius of SIMONIC apps to a certain distance $(5 \mathrm{~m}, 10 \mathrm{~m}, 15 \mathrm{~m}, 20 \mathrm{~m})$, and we

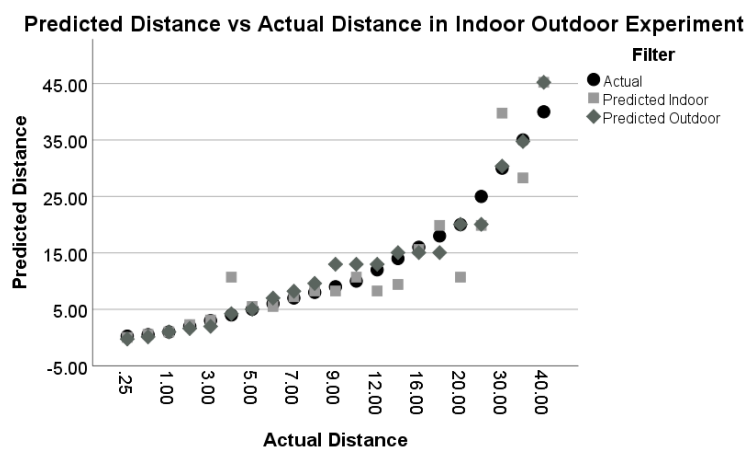

Figure 11. Predicted Distance vs. Actual Distance from $0.25 \mathrm{~m}-40 \mathrm{~m}$ in Indoor and Outdoor.

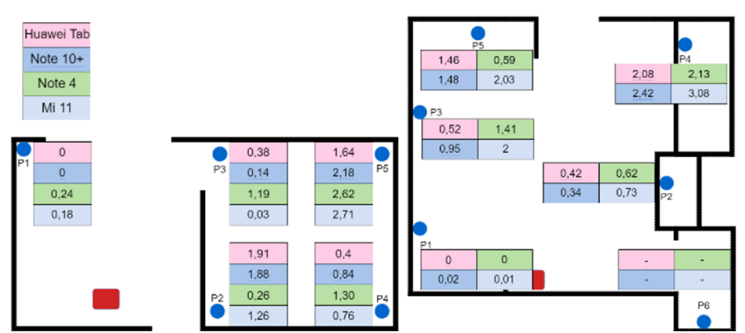

(a)

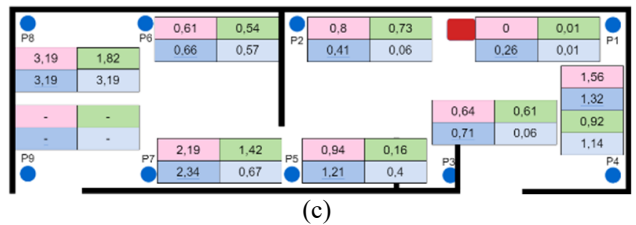

Figure 12. Error Value in Various Quarantine Room: (a) Small Room $\left(18 \mathrm{~m}^{2}\right)$, (b) Apartment Room $\left(24 \mathrm{~m}^{2}\right)$, (c) Pavilion Room $\left(27 \mathrm{~m}^{2}\right)$. 
place the wristband in each range for each measurement. We conduct measurements indoor and outdoor which are crowded enough using six different smartphone types. Tables 2 and 3 describe the experiment result.

Precision is defined as a percentage value of correct notification alert when any SIMONIC wristband approaches the smartphones in all scanning radius $(5 \mathrm{~m}$, $10 \mathrm{~m}, 15 \mathrm{~m}$, and $20 \mathrm{~m}$ ) compared to all notification alerts that pop up right or wrong. This means how precise the pop-ups notification alert represents the correct conditions of wristband being in scanning radius. The recall represents the correct notification alert when any SIMONIC wristband approaches the smartphones in all scanning radius $(5 \mathrm{~m}, 10 \mathrm{~m}, 15 \mathrm{~m}$, and $20 \mathrm{~m})$ compared to the actual condition of whether the notification alert pops up or not. This means how good a notification alert will pop up when the wristband is in the scanning radius. Accuracy shows that all notification alerts pop up, whether right or wrong, compared to all system behavior (true condition/false condition). This means how much the notification alerts pop up, whether right or wrong, compared to all system behavior. The harmonic mean of precision and recall is defined as F1-score.

TABLE 2

PreCision, ReCALl, ACCURACY, F1-SCORE OF SIMONIC NOTIFICATION FOR SOCIAL DISTANCING FEATURE MEASURE WITH DIFFERENT SMARTPHONES

\begin{tabular}{|c|c|c|c|c|c|}
\hline & Device & Precision & Recall & Accuracy & F1-Score \\
\hline \multirow{6}{*}{ Indoor } & $\begin{array}{l}\text { Samsung } \\
\text { Note } 10\end{array}$ & $81.11 \%$ & $73.83 \%$ & $67.91 \%$ & $74.19 \%$ \\
\hline & $\begin{array}{l}\text { Huawei } \\
\text { Tab }\end{array}$ & $85.41 \%$ & $88.10 \%$ & $81.24 \%$ & $86.35 \%$ \\
\hline & \begin{tabular}{|l|} 
Redmi \\
$8 \mathrm{~A}$ \\
\end{tabular} & $85.96 \%$ & $79.59 \%$ & $74.97 \%$ & $81.97 \%$ \\
\hline & \begin{tabular}{|l|} 
Samsung \\
A10
\end{tabular} & $91.39 \%$ & $76.79 \%$ & $76.68 \%$ & $80.13 \%$ \\
\hline & \begin{tabular}{|l|} 
Samsung \\
A50
\end{tabular} & $74.25 \%$ & $85.83 \%$ & $72.67 \%$ & $79.45 \%$ \\
\hline & \begin{tabular}{|l|} 
Samsung \\
S20FE
\end{tabular} & $88.51 \%$ & $85.14 \%$ & $81.16 \%$ & $86.03 \%$ \\
\hline \multirow{6}{*}{ Outdoor } & $\begin{array}{l}\text { Samsung } \\
\text { Note } 10\end{array}$ & $87.59 \%$ & $75.91 \%$ & $74.00 \%$ & $78.12 \%$ \\
\hline & $\begin{array}{l}\text { Huawei } \\
\text { Tab }\end{array}$ & $91.44 \%$ & $89.64 \%$ & $85.40 \%$ & $89.59 \%$ \\
\hline & \begin{tabular}{|l} 
Redmi \\
$8 \mathrm{~A}$
\end{tabular} & $87.07 \%$ & $82.20 \%$ & $77.16 \%$ & $83.65 \%$ \\
\hline & $\begin{array}{l}\text { Samsung } \\
\text { A10 }\end{array}$ & $87.83 \%$ & $80.95 \%$ & $77.22 \%$ & $82.05 \%$ \\
\hline & $\begin{array}{l}\text { Samsung } \\
\text { A50 }\end{array}$ & $91.05 \%$ & $77.99 \%$ & $77.66 \%$ & $80.89 \%$ \\
\hline & \begin{tabular}{|l|} 
Samsung \\
S20FE
\end{tabular} & $91.45 \%$ & $87.82 \%$ & $85.17 \%$ & $87.96 \%$ \\
\hline
\end{tabular}

TABLE 3

Precision, ReCAll, ACCURACy, F1-Score of SIMONIC NOTIFICATION FOR SOCIAL DistanCING FEATURE MEASURE IN DIFFERENT DISTANCE

\begin{tabular}{|c|r|c|c|c|c|}
\cline { 2 - 6 } \multicolumn{1}{c|}{} & Range & Precision & Recall & Accuracy & F1-Score \\
\hline \multirow{4}{*}{ Indoor } & $5 \mathrm{~m}$ & $89.29 \%$ & $64.76 \%$ & $73.61 \%$ & $73.28 \%$ \\
\cline { 2 - 6 } & $10 \mathrm{~m}$ & $82.88 \%$ & $69.17 \%$ & $67.39 \%$ & $74.46 \%$ \\
\cline { 2 - 6 } & $15 \mathrm{~m}$ & $81.79 \%$ & $93.01 \%$ & $78.33 \%$ & $86.81 \%$ \\
\cline { 2 - 6 } & $20 \mathrm{~m}$ & $83.80 \%$ & $99.24 \%$ & $83.75 \%$ & $90.86 \%$ \\
\hline \multirow{5}{*}{ Outdoor } & $5 \mathrm{~m}$ & $100.00 \%$ & $57.14 \%$ & $75.00 \%$ & $72.02 \%$ \\
\cline { 2 - 6 } & $10 \mathrm{~m}$ & $93.35 \%$ & $79.17 \%$ & $81.37 \%$ & $85.26 \%$ \\
\cline { 2 - 6 } & $15 \mathrm{~m}$ & $80.79 \%$ & $94.12 \%$ & $78.03 \%$ & $86.88 \%$ \\
\cline { 2 - 6 } & $20 \mathrm{~m}$ & $83.49 \%$ & $99.24 \%$ & $83.33 \%$ & $90.67 \%$ \\
\hline
\end{tabular}

\section{Geofence Performance}

We conducted field trials to study and validate the geofence performance of this system. The trials were conducted on five volunteers who live in several areas in Bandung, West Java. Following how the system works, each volunteer is in their respective residence, considered as a quarantine place. The registration process is monitored online via video call by the supervisor/officer to minimize direct contact with quarantine patients. Each volunteer installs and activates the SIMONIC application through the Playstore, activates the GPS, puts on a wristband, and the supervisor activates the system. Volunteers set the scanning interval and detection radius. Volunteers then calibrate the distance explained at point III.B and collect location data by placing the smartphone in the middle of the room for five minutes. The system then detects the location coordinates, and the supervisor creates a geofence from the tracking system according to the required size.

Volunteers wore wristbands and were monitored for three days and three nights to measure the performance of the geofence system. We measure several parameters as listed in Table 4. During the monitoring period, volunteers will carry out daily activities instead of staying inside the quarantine room. We calculated the system readings at night when the volunteers were sleeping and in the quarantine room for the false alarm rate parameter. To determine the effectiveness of the in/out geofence alarm, we observed when volunteers left the house for work or other activities. Volunteers will report activities within three days when they are out of the quarantine room.

Precision is defined as the ratio of the patient's position that can be correctly recognized by the system in total, whether inside or outside the quarantine rooms, to the total prediction of the correct condition. Precision represents the rightness of the system in predicting the patient location. A recall is defined as the ratio of the patient's position that the system can correctly recognize in total to the total correct condition. The recall represents the system's success in detecting the correct patient location. Accuracy is the ratio between the number of correct conditions in which the system detects the patients inside or outside the quarantine room and the total number of cases. Accuracy counts how well the predictions can predict the correct condition of the

TABLE 4

PreCision, RECALL, ACCURACY, F1-SCORE OF SIMONIC FOR MEASURING THE GEOFENCE PERFORMANCE

\begin{tabular}{|c|c|c|c|c|c|c|}
\hline Loc* & Device* & $\begin{array}{l}\text { Room } \\
\text { Size }\end{array}$ & Prec & Rec & Acc & $\begin{array}{c}\text { F1- } \\
\text { Score }\end{array}$ \\
\hline 1 & $\mathrm{a}$ & $18 \mathrm{~m}^{2}$ & $99 \%$ & $98 \%$ & $97 \%$ & $98 \%$ \\
\hline 2 & $\mathrm{~b}$ & $60 \mathrm{~m}^{2}$ & $95 \%$ & $98 \%$ & $94 \%$ & $96 \%$ \\
\hline 3 & $\mathrm{c}$ & $72 \mathrm{~m}^{2}$ & $97 \%$ & $96 \%$ & $98 \%$ & $97 \%$ \\
\hline 4 & $\mathrm{~d}$ & $45 \mathrm{~m}^{2}$ & $97 \%$ & $98 \%$ & $97 \%$ & $98 \%$ \\
\hline 5 & $\mathrm{e}$ & $50 \mathrm{~m}^{2}$ & $94 \%$ & $99 \%$ & $95 \%$ & $97 \%$ \\
\hline & & & & \multicolumn{3}{|c|}{$\begin{array}{l}\text { 1*: Cipendeuy, Bandung Barat. } \\
\text { 2*: Cibiru, Kab. Bandung. } \\
\text { 3*: Sariwangi, Kota Bandung. } \\
\text { 4*: Margaasih, Cimahi. } \\
\text { 5*: Cipagalo, Kab. Bandung. } \\
\text { a*: Vivo Y17 } \\
\text { b*: Infinix Smart 3+ } \\
\text { c*: Samsung Galazy A30s } \\
\text { d*: Redmi Note 8 } \\
\text { e*: Smartfren }\end{array}$} \\
\hline
\end{tabular}


system. The harmonic mean of precision and recall, in this case, is defined as F1-score.

\section{Discussion}

The first experiment in this paper is to conduct an empirical study for the communication performance between wristband and smartphone and the wristband battery durability. The results of this study indicate that wristband-to-smartphone communication can be done well inside the room. If it is done in an indoor LOS room, as depicted in Figure 6(a), communication can still be carried out up to a distance of 20 meters even though the RSSI has decreased quite a lot. However, if the wristband and smartphone are placed in different rooms, communication difficulties cause the wristband BLE signal to be unreadable by the smartphone, as can be seen in Figure 12(b) for P6 and Figure 12(c) for P9. Therefore, we recommend always keeping the wristband close to the smartphone in the quarantine process and not placing the smartphone in a different room.

The following empirical study establishes communication-based on the scanning interval set up in the smartphone. Due to battery consumption and the calculation from Figures 7 and 9, scanning interval per seven seconds is the most suitable implementation in this system, and it can provide one communication establishment in a minute. Then we come to the result of the battery durability test. Considering several things that are the results of experiments and analyses in chapter III.A, we decided to use the advertising interval of 7000 ms and the $0 \mathrm{dBm}$ power module to apply this wearable device.

The second experiment in this paper is to measure the notification for social distancing performance. However, we measure the distance measurement performance first. We measure system performance based on distance on various quarantine rooms, as seen in Figure 5, with quiet environmental conditions and not many people passing by. The results are represented in Figure 12 with the most significant error value (predicted distance vs. actual distance) $=3.19 \mathrm{~m}$.

After that, we conducted experiments to measure the performance of the notification feature for social distancing in pretty much crowded indoor and outdoor environments. From Table 2, we can conclude that the average precision for all range measurements and smartphones in indoor and outdoor areas is above $81 \%$, with an average value of $86.92 \%$. It means that the system is precise in generating the notification for social distancing alerts. For the recall, we get most of the values above $75 \%$. The average value is $81.98 \%$, which means the goodness of popping up notification alerts when the wristband is in scanning radius is still good enough, only for Samsung note 4 , which is $73 \%$. Meanwhile, we get the lowest value at $67.91 \%$ in the accuracy and the average value at $77.60 \%$. This means the notification alerts pop-up is low compared to what it should be.

Furthermore, we get F1-score values above $74.19 \%$ for the harmonic means of precision and recall. When we look closer at the scanning radius setting detail, the result is depicted in Table 3. We can see that the pop-up notification alert's precision is high for the $5 \mathrm{~m}$ and $10 \mathrm{~m}$ compared to $15 \mathrm{~m}$ and $20 \mathrm{~m}$, whereas the recall value in $5 \mathrm{~m}$ and $10 \mathrm{~m}$ indoor and outdoor is low compared to 15 $\mathrm{m}$ and $20 \mathrm{~m}$. This means that even though the system is not optimal in generating alerts for social distancing at a distance of $5-10 \mathrm{~m}$, the alert produced is precise enough to represent the correct conditions of wristband being in scanning radius.

Moreover, for the scanning radius $15 \mathrm{~m}$ and $20 \mathrm{~m}$, both precision and recall are in high percentage value. This means that the system optimally generates alerts for social distancing, and the alert produced is precise enough to represent the correct conditions of wristband being in scanning radius. For this result, the low average recall value in $5 \mathrm{~m}$ and $10 \mathrm{~m}$ could be affected by the RSSI value, which the distance calculation depends on. The environmental condition greatly influences the RSSI value. This causes the RSSI reading to be not stable enough to be converted to a distance and affects the performance of reading the location of the wristband.

The third experiment is to measure the geofence performance. From Table 4, we can see that the experiment was done by five different persons, using different smartphone types, conducted in different areas in Bandung and different types of rooms. From the values of precision, recall, accuracy, and F1-score that we get, which all the average are above $95 \%$, it can conclude that the geofence system built using the Traccar application can function properly to create geofences and can provide reasonably accurate information about the position of patients who are in quarantine.

\section{CONCLUSION}

In this study, we proposed an IoT-based quarantine monitoring system, consisting of a low-cost wristband, low complexity, and reusable design connected to a smartphone with android apps installed. We conducted some experiments to validate the system. In terms of wristband-to-smartphone communication performance, the experiment result shows that the communication can be maintained well indoors without any wall barrier in between. Due to the consideration of battery consumption, scanning interval per $7 \mathrm{~s}$ is the most suitable implementation in this system, and it is capable of providing one communication establishment in a minute. Based on the experiment result, the best advertising interval setting of wristband considering the Li-Batt power is at $7 \mathrm{~s}$. The notification for social distancing performance is measured using six different smartphones in an indoor and outdoor environment. Overall, the system is precise in generating the notification for social distancing alerts with an average precision value of $86.92 \%$. The amount of notification alerts produced when the wristband is in scanning radius is still good enough, with a recall average value of 81.98 $\%$. For the geofence performance, an experiment is conducted by five different persons, smartphones, areas, and room types. With average values above $95 \%$ for precision, recall, accuracy, and F1-score, we can conclude that the geofence system built using the Traccar application can function properly to create geofences and provide fairly accurate information about the position of patients who are in quarantine. 


\section{ACKNOWLEDGMENT}

This research is fully funded and supported by Indonesia Endowment Fund for Education (LPDP).

\section{REFERENCES}

[1] "World health organization." https://www.who.int/ (accessed Dec. 28, 2021).

[2] H. Ryu, A. Abulali, and S. Lee, "Assessing the effectiveness of isolation and contact-tracing interventions for early transmission dynamics of covid-19 in South Korea," IEEE Access, vol. 9, pp. 41456-41467, 2021, doi: 10.1109/ACCESS.2021.3064371.

[3] "Quarantine in Japan." https://quarantine-japan.com/ (accessed Dec. 28, 2021).

[4] G. Jaswal, R. Bharadwaj, K. Tiwari, D. Thapar, P. Goyal, and A. Nigam, "AI-biometric-driven smartphone app for strict postCOVID home quarantine management," IEEE Consum. Electron. Mag., vol. 10, no. 3, pp. 49-55, 2021, doi: 10.1109/MCE.2020.3039035.

[5] J. Tan, E. Sumpena, W. Zhuo, Z. Zhao, M. Liu, and S. G. Chan, "IoT geofencing for COVID-19 home quarantine enforcement," IEEE Internet of Things Mag., vol. 3, no. 3, pp. 24-29, Sep. 2020, doi: 10.1109/IOTM.0001.2000097.

[6] Y. C. Hou, M. Z. Baharuddin, S. Yussof, and S. Dzulkifly, "Social distancing detection with deep learning model," 2020 8th Int. Conf. Inf. Technol. Multimedia, 2020, pp. 334-338, doi: 10.1109/ICIMU49871.2020.9243478.

[7] M. E. Rusli, S. Yussof, M. Ali, and A. A. Abobakr Hassan, "MySD: A smart social distancing monitoring system," $20208 \mathrm{th}$ Int. Conf. Inf. Technol. Multimedia, 2020, pp. 399-403, doi: 10.1109/ICIMU49871.2020.9243569.

[8] A. Narzullaev, Z. Muminov, and M. Narzullaev, "Contact tracing of infectious diseases using Wi-Fi signals and machine learning classification," 2020 IEEE 2nd Int. Conf. Artif. Intell. Eng. Technol., 2020, doi: 10.1109/IICAIET49801.2020.9257812.

[9] O. Ruan, T. Liu, and D. Zhou, "Efficient and privacy-preserving of COVID-19 contact tracing scheme," 2020 Int. Conf. Comput. Sci. Manag. Technol., 2020, pp. 105-108, 2020, doi: 10.1109/ICCSMT51754.2020.00028.

[10] W. Tan and J. Liu, "Application of face recognition in tracing COVID-19 fever patients and close contacts," 2020 19th IEEE Int. Conf. Mach. Learn. Appl., 2020, pp. 1112-1116, doi 10.1109/ICMLA51294.2020.00179.

[11] C. Sonia Villamizar, G. Edwar Jacinto, and A. Holman Montiel, "Design of an electronic bracelet for remote surveillance of people deprived of their freedom in Colombia," Int. J. Appl. Eng. Res., vol. 12, no. 24, pp. 15452-15457, 2017.

[12] M. F. Monir, A. H. Chowdhury, R. Anzum, and M. A. Amin, "IoT enabled geofencing for covid-19 home quarantine," 20218 th Int. Conf. Comput. Commun. Eng., 2021, pp. 373-378, doi: 10.1109/ICCCE50029.2021.9467204.

[13] E. R. Pratama, F. Renaldi, F. R. Umbara, and E. C. Djamal, "Geofencing technology in monitoring of geriatric patients suffering from dementia and alzheimer," 2020 3rd Int. Conf.
Comput. Informatics Eng., 2020, pp. 106-111, doi: 10.1109/IC2IE50715.2020.9274637.

[14] J. Helmy and A. Helmy, "Demo abstract: Alzimio: A mobile app with geofencing, activity-recognition and safety features for dementia patients," 2017 IEEE Conf. Comput. Commun. Workshop, 2017, pp. 994-995, doi: 10.1109/INFCOMW.2017.8116527.

[15] B. Nayak, P. S. Mugali, B. R. Rao, S. Sindhava, D. N. Disha, and K. S. Swarnalatha, "Geofencing-based accident avoidance notification for road safety," Emerg. Res. Comput. Information, Commun. Appl., 2019, vol. 906, pp. 379-386. doi: 10.1007/978981-13-6001-5 30

[16] F. Besoain, A. Perez-Navarro, C. J. Aviñó, J. A. Caylà, N. A. Barriga, and P. G. de Olalla, "Prevention of HIV and other sexually transmitted infections by geofencing and contextualized messages with a gamified app, UBESAFE: Design and creation study," J. Medical Internet Res. Mhealth Uhealth, vol. 8, no. 3, 2020, Art. no. e1456, doi: 10.2196/14568.

[17] S. Suryadi, E. Kurniawan, H. Adinanta, B. H. Sirenden, J. A Prakosa, and P. Purwowibowo, "On the comparison of social distancing violation detectors with graphical processing unit support," 2020 Int. Conf. Radar, Antenna, Microwave, Electron. Telecommun., 2020, pp. 337-342, doi: 10.1109/ICRAMET51080.2020.9298574

[18] M. Sharma, "Open-CV social distancing intelligent system," 2020 2nd Int. Conf. Adv. Comput. Commun. Control Networking, 2020, pp. 972-975, doi: 10.1109/ICACCCN51052.2020.9362920.

[19] P. Somaldo, F. A. Ferdiansyah, G. Jati, and W. Jatmiko, "Developing smart COVID-19 social distancing surveillance drone using YOLO implemented in robot operating system simulation environment," 2020 IEEE 8th R10 Humanitarian Technol. Conf., 2020, doi: 10.1109/R10 HTC49770.2020.9357040.

[20] A. Gad, G. Elbary, M. Alkhedher, and M. Ghazal, "Vision-based approach for automated social distance violators detection," 2020 Int. Conf. Innov. Intell. Informatics Comput. Technol., 2020, doi: 10.1109/3ICT51146.2020.9311969.

[21] F. A. A. Naqiyuddin, W. Mansor, N. M. Sallehuddin, M. N. S. M. Johari, M. A. S. Shazlan, and A. N. Bakar, "Wearable social distancing detection system," 2020 IEEE Int. RF Microw. Conf., 2020, doi: 10.1109/RFM50841.2020.9344786.

[22] "Traccar - modern GPS tracking platform.' https://www.traccar.org/ (accessed Dec. 28, 2021).

[23] "Trial Methodologies." https://github.com/opentracecommunity/opentrace-calibration/blob/master/Trial Methodologies.md (accessed Dec. 28, 2021).

[24] Z. Ozdemir and B. Tugrul, "Geofencing on the real-time GPS tracking system and improving GPS accuracy with moving average, Kalman filter and logistic regression analysis," $20193 \mathrm{rd}$ Int. Symp. Multidiscip. Stud. Innov. Technol., 2019, doi: 10.1109/ISMSIT.2019.8932766.

[25] P. Chunhakam, P. Pummarin, P. Jeen-Im, P. Wardkien, P. Wisartpong, and K. Lertteerada, "GPS position predicting system by Kalman filter with velocity from OBD and direction from magnetometer," 2021 9th Int. Electr. Eng. Congr., 2021, pp. 444 447, doi: 10.1109/iEECON51072.2021.9440239. 